\title{
The change of the properties of the turning process by introducing vibrations in the cutting zone
}

\author{
Vilor Zakovorotny, and Valery Gvindjiliya* \\ Don State Technical University, 344000 Rostov-on-Don Gagarin sq. 1, Russian Federation
}

\begin{abstract}
The results of the research of the vibrations influence are raising in the cutting zone on the output properties of the cutting process are represented. The results of the mathematical modeling of the dynamic cutting system are represented in which the controlling oscillatory displacements are raised. The system is considered as the unity subsystem of the tool and the detail are interacting through the processing. It is shows that the additional vibrations transform in the geometry topology of the forming surface as the result of the complex nonlinear interaction of the oscillations and the forces. The additional oscillations can cause as improvement of accuracy, quality and intensity of the tool wearing as deterioration of output process characteristics depending on the parameters of the vibration (amplitude, frequency and orientation). The results of the research of the oscillations influence on the quality of the surface and the power of the irreversible transformations which characterize the cutting process state are represented.
\end{abstract}

\section{Introduction}

The study of the vibrations influence on the output characteristics of the cutting process is the traditional in the research of the processing on the cutting machines [1 - 5]. The forced oscillations and the self-oscillations are generated by the interact of the dynamic subsystems from outside the cutting tool and the work piece with the cutting process are analyzed. The interaction is represented as the dynamic link is formed by the cutting process. In this the oscillations in the wide frequency range of tens $\mathrm{Hz}$ to tens $\mathrm{kHz}$ are analyzed. The researches of the oscillations influence on the cutting process are largely executed experimentally. Their purpose is clarification of the oscillations influence on the intensity of the wearing of the cutting tool and the accuracy and the quality of the parts manufacturing. At the same time, it is noted that the increase or decrease wearing of the cutting tool is possible depending on the parameters oscillations (frequency, amplitude and orientation). It is noted that the increase of the surface quality of the one quality is possible at the defined parameters of the oscillations [6 - 10]. It is necessary to consider the properties of the dynamic cutting tool for clarification of the oscillation influence [11 - 21]. The problems of the stability [11-14] and the forming of the attracting sets of the

*Corresponding author: sinedden@yandex.ru 
deformational displacement [15-18] and their bifurcation in parametric space are considered [19-22]. The conditions of the parametrical self-excitation are analyzed [23]. In recent years the work about the dynamic perturbation systems are published. The perturbations are determined by the spindle beats group and the kinematic perturbations [ 24 - 26]. In works $[27,28]$ take into consideration that the influencing reaction outside the cutting process on the oscillations are excited by the additional source. The self-resonance systems [29] are suggested to provide the conditions of the self-excitation take into account the reaction outside the cutting process. The regularity of the transformation of the vibration in the deformational displacement trajectories and the power of the irreversible transformations in cutting zone take into account nonlinear cutting effects are stated on the basis of the mathematical models and the numeric experiments are elaborated by authors. It supplements knowledge of the vibration control of the cutting process.

\section{The mathematical modeling of the influence oscillations on the cutting process}

In modeling let's use the mathematical models are received early take into account the setting parameters of the oscillation displacement are introduced in cutting zone $[24,25,30,31]$. Then

$$
m \frac{d^{2} X}{d t^{2}}+h \frac{d X}{d t}+c X=F\left(X, t_{P}^{(0)}, S_{P}^{(0)}, V_{3}, U\right)+\Phi\left(X, t_{P}^{(0)}, S_{P}^{(0)}, V_{0}, U\right)
$$

where $m, h, c$ - symmetric and positive-definite matrix of the inertial, dissipation and elastic characteristics of the tool subsystem of the dimension $3 \otimes 3$; $X=\left\{X_{1}, X_{2}, X_{3}\right\}^{T} \in \mathfrak{R}^{(3)}$ - vector of the elastic deformational displacement of the tool tip relative to the support system of the machine; $F=\left\{F_{1}, F_{2}, F_{3}\right\}^{T} \in \mathfrak{R}^{(3)}$, $\Phi=\left\{\Phi_{1}, \Phi_{2}, \Phi_{3}\right\}^{T} \in \mathfrak{R}^{(3)}$ - forces vector are acting in conformity with front and back the tool surface are represented in the elastic deformational displacement $(X)$, setting and constant of the technological regimes (cutting depth $t_{P}^{(0)}$, turnover supply $S_{P}^{(0)}$ and cutting speed $V_{3}$ ) and the external vibrations ( $U$ ).In (1) $\mathfrak{R}^{(3)}$ is Euclidean space in which: $X_{1}$ is the direction normal to the preparation axis; $X_{2}$ - the opposite direction to the speed longitudinal feeding; $X_{3}$ - directional of the cutting speed. The longitudinal turning of the non-deformable detail is considered. The main corner in a plane of the cutting tool equal $\pi / 2$. Therefore the main cutting blade coincide with axis $X_{1}$.

The external influences are considered as the setting functions $U(t)=U_{0} \sin \Omega t$ are orientating to one of the directions of the space $\mathfrak{R}^{(3)}$. The following ideas continuation from works $[24,25]$ are used to representation of the forces in the coordinates of the state space and the external influences. For forces $F$

$$
\left\{\begin{array}{c}
T_{1} d F_{1} / d t+F_{1}=\rho \chi_{1}\left\{1+\mu \exp \left[-\zeta\left(V_{3}-d X_{3} / d t\right)\right]\right\}\left[t_{P}^{(0)}-X_{1}\right] S_{P}(t) \\
T_{2} d F_{2} / d t+F_{2}=\rho \chi_{2}\left\{1+\mu \exp \left[-\zeta\left(V_{3}-d X_{3} / d t\right)\right]\right\}\left[t_{P}^{(0)}-X_{1}\right] S_{P}(t) ; \\
T_{3} d F_{3} / d t+F_{3}=\rho \chi_{3}\left\{1+\mu \exp \left[-\zeta\left(V_{3}-d X_{3} / d t\right)\right]\right\}\left[t_{P}^{(0)}-X_{1}\right] S_{P}(t),
\end{array}\right.
$$


where $T_{i}, i=1,2,3$ - time constant; $\mu$ - the coefficient is determining correlation of the forces in the area of the low and high speeds; $\zeta$ - the coefficient is determining the decrease of the forces with the increase of the speed; $V_{3}$ - cutting speed; $\rho$ - shaving pressure on the front surface of the tool; $S_{P}(t)=\int_{t-T}^{t}\left[V_{2}-d X_{2} / d t\right] d t=S_{P}^{(0)}-X_{2}(t)+X_{2}(t-T)$ - the operator of the formation of the turnover feeding. For forces

$$
\left\{\begin{array}{c}
\Phi_{1}=\rho_{0}\left\{\int_{t-T}^{t}\left[V_{2}-d X_{2} / d t\right] d t\right\} \exp \left[\alpha_{0}\left(d X_{1} / d t\right)\right] ; \\
\Phi_{2}=\rho_{0}\left[t_{P}^{(0)}-X_{1}\right] \exp \alpha_{0}\left[\left(V_{2}-d X_{2} / d t\right] ;\right. \\
\Phi_{3}=k_{T}\left(\Phi_{1}+\Phi_{2}\right),
\end{array}\right.
$$

where $\rho_{0}$ - the forces are reduction to length of the contact; $\alpha_{0}$ - the coefficient of the force increase; $k_{T}$ - friction coefficient; $V_{2}$ - speed of the longitudinal support. In the systems (2), (3) $t_{P}^{(0)}, S_{P}^{(0)}, V_{2}, V_{3}$ - the constant values of the external influences are set by the trajectories of the executive elements of the machine. The external vibrating influences are introduced additional to the value $t_{P}^{(0)}$ for example in direction $X_{1}$.

\section{The influence of the additional oscillation on the properties of the cutting process}

The surface geometry depends on the trajectories of the trajectories vibrating displacement, the elastic deformational of the tool and the executive elements are set by the control. It is necessary to find out the depending of the deformational displacement of the tool tip on the parameters and the additional oscillations entered in cutting zone with the estimation of the oscillations influence on the geometry topology. The regularity of the influence of the oscillations depend on their frequency range is depending on the fundamental frequencies of the tool subsystem without the reaction outside the cutting process. Let's disclose the property of the transformation of the additional oscillations in the deformational displacement as example of the longitude turning with technological regimes: $S_{P}^{(0)}=0,1 \mathrm{~mm}, t_{P}^{(0)}=2,5 \mathrm{~mm}, V_{3}=1,5 \mathrm{~m} / \mathrm{s}$. Let's put: the axis of the rigid ellipsoid of the tool subsystem coincide with the space axis $\mathfrak{R}^{(3)}$; The parameters of the tool subsystem:

$c_{1,0}=3000 \mathrm{~kg} / \mathrm{mm}, c_{2,0}=1000 \mathrm{~kg} / \mathrm{mm}, c_{3,0}=600 \mathrm{~kg} / \mathrm{mm} ; h_{0}=5,0 \mathrm{~kg} \cdot \mathrm{s} / \mathrm{mm}$; $m_{0}=0,025 \mathrm{~kg} \cdot \mathrm{s}^{2} / \mathrm{mm}$. Let's consider $T_{1}=T_{3}=0,5 T_{2}=T$. The fundamental frequency of the tool subsystem: $\Omega_{1}=350 \mathrm{~s}^{-1}, \Omega_{2}=200 \mathrm{~s}^{-1}, \Omega_{3}=120 \mathrm{~s}^{-1}$. The parameters of the dynamic link are showed in Table. 1.

Table 1. The parameters of the dynamic link of the cutting process

\begin{tabular}{|c|c|c|c|c|c|}
\hline $\boldsymbol{\rho}_{\mathbf{0}, \mathbf{0}}, \mathbf{k g} / \mathbf{m m}$ & $\zeta, \boldsymbol{s} / \mathbf{m m}$ & $\alpha_{\mathbf{0}}, \mathbf{s} / \mathbf{m m}$ & $\boldsymbol{T}, \boldsymbol{s}$ & $\boldsymbol{\rho}_{\mathbf{0}}, \mathbf{k g} / \mathbf{m m}$ & $\boldsymbol{\mu}$ \\
\hline $150-1000$ & $1,0-5,0$ & $(20-50)$ & $(0,001-0,01)$ & 2,0 & 0,5 \\
\hline
\end{tabular}


It is expedient to consider three frequency ranges for studying of the influence of the oscillations on the deformational displacements: first - $\Omega^{(1)}\langle 50 \mathrm{~Hz}$; second $\Omega^{(2)} \in(50,0,500,0) H z$; third - $\Omega^{(3)}>500,0 H z$. The introducing vibrations cause «slow» shifts of the equilibrium point in range $\Omega^{(1)}\langle 50 \mathrm{~Hz}$ and in direction of the oscillations on the axis $X_{1}$ from the allowance value. The vibrations in direction $X_{2}$ and $X_{3}$ is orthogonal to the forming surface. In this case the changes of the shape-forming trajectories is determined by the deformational displacement in direction $X_{1}$. The oscillations including the deformational displacements lead to slow periodic changes of the variational equations relative to stationary trajectories. At the oscillations in direction $X_{1}$ the most sensitivity of the dynamic system to change her properties is observed since the contact length of the main cutting blade with workpiece change. Therefore, the periodic changes of the system parameters along the trajectory of the «slow» motions are observed. The transformations of the attractive sets like the limit cycles through cascade of the bifurcation of the doubling period to the chaotic oscillations (fig.1 «a») are possible in depending on the roots distant of the characteristic polynom of the linearized variational equation along the trajectory. All stages of these transformations periodically repeat. In the frequency range $\Omega^{(2)}$ at first the parametrical effect including the periodic loss of the stability with increase of the rotation frequency are observed. Secondly the effect of the interaction inputting oscillations with self-oscillations is observed it causes the synchronization of some periodical motions and elimination other and their asynchronous interaction. Thirdly the self-excitation of the fractional order (fig. $1 《 b »)$ is possible with some parameters subsystem of the tool and the dynamic link is formed in the region of the contact of the tool backside with workpiece. In the case the oscillatory displacements with frequency $250 \mathrm{~Hz}$ cause the deformational displacements in direction $X_{1}$ with frequency $4 \mathrm{~Hz}$. This effect causes the sinuosity formation on the detail surface. It is important to emphasize that conditions in which we observe the strengthening of the oscillations whose motions are orthogonal to the surface roughness formed that is to the effect of the vibrational stabilization of the relief formed by the cutting process are possible. In this case the quality of the surface increases through the oscillations.

In the range frequency $\Omega^{(3)}$ input oscillations lie outside of the bandwidth of the oscillation circuits. They periodically change interactions of the tool backside with detail, forming the dynamic displacement of the equilibrium. In the contact region of the frontside with cutting region the cyclically stress states arise which change the parameters of the dynamic characteristic of the cutting process together after averaging over the periods of the inputting oscillations. It is influence on the system stability. In fig. 1 «c» the system loses stability at introduction of the oscillations and the self-oscillations are formed. The average value of the deformational displacement increases and consequently the diameter of the work piece increases. Through the self-oscillations the periodically displacements on the surface of the detail form which topology is similar considered in work [24]. The formation of the cyclically tension both in the region of the elastic and the plasticity region is observed with oscillations in direction of the cutting speed (fig. 1 «d») in the region of the contact of the front surface of the tool in cutting zone. It leads to reduction of the integrate over periods of the forces fluctuations are perceived by the tool and the detail that cause reduction of the elastic deformational displacements that is reduction of the detail diameter. If the speed of the oscillation displacements with elastic deformational approaches or becomes the higher of the cutting speed, then the effect of the reduction of the integrate over forces is observed particularly visible. These data show that the transformation entering oscillations in the cutting zone contradiction influence on the 
cutting process and this influence is the result of the complex nonlinear transformations of the oscillations of the dynamic cutting system. And the influences of the oscillations on the irreversible transformations of the energy of the mechanical system in zones of the contact of the tool side with zone of the process are also contradiction. Therefore, the influence of the oscillations on the intensity of the wearing of the cutting tool is contradiction.
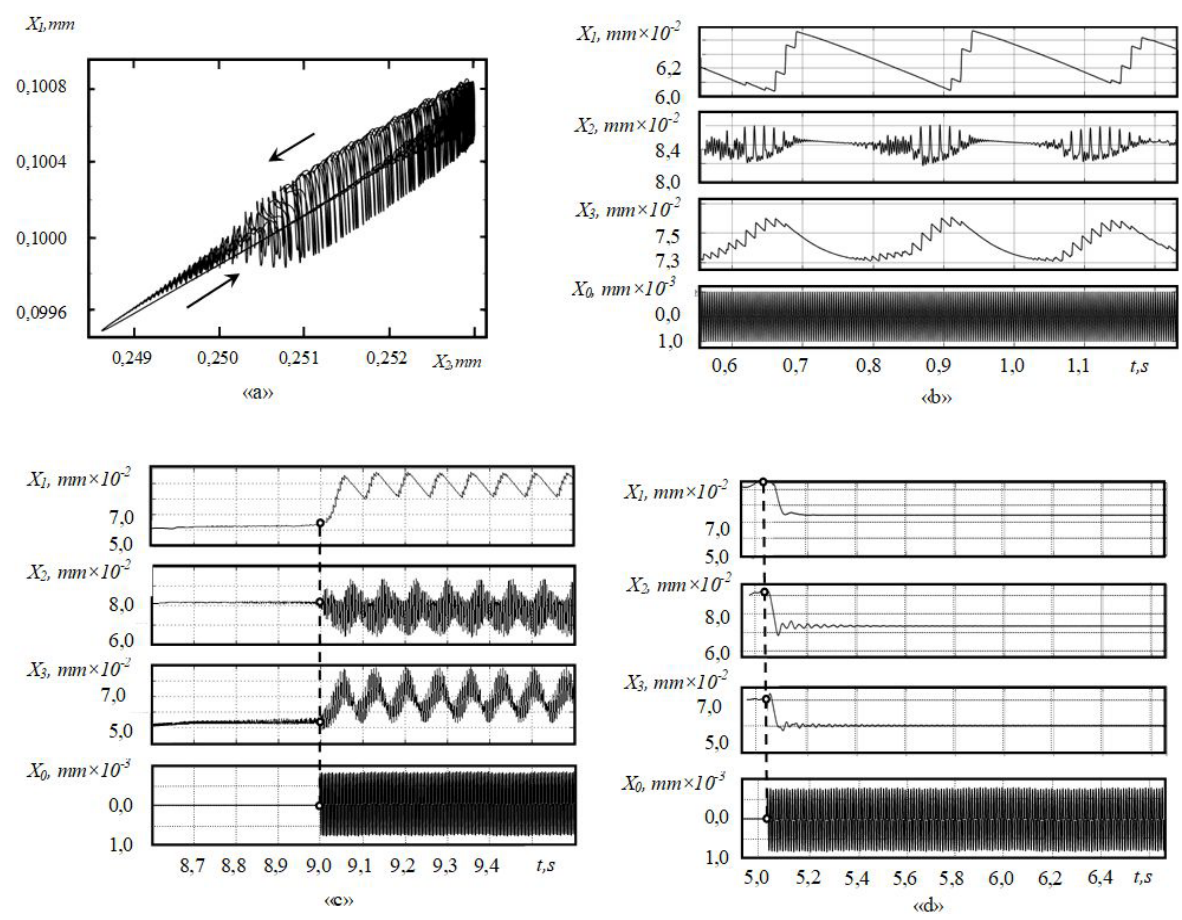

Fig. 1. Examples of the changes of tip tool deformations in depending on the entered oscillations: a trajectories of the deformation displacement in plane $X_{1}, X_{2}$ in the frequency range $\Omega^{(1)} ; \mathrm{b}-$ examples of the self-excitation of the fractional order with oscillations excitation in direction $X_{1}$ in the frequency range $\Omega^{(2)} ; \mathrm{c}$-change of the properties of the deformational displacement with oscillations excitation in direction $X_{2} ; \mathrm{d}$ - change of the properties of the deformational displacements with excitation of the oscillations in direction $X_{3}$

\section{Conclusion}

The dynamic cutting system is the complex nonlinear transformer of all perturbations are acting her including entering oscillations in cutting zone. In depending on the parameters of the oscillations (amplitude, frequency and direction) the oscillations can both worsen the output properties of the cutting process and improve them. This thesis relates to the parameters of the topology is formed by the cutting process and the intensity of the wearing of the tool. Apparently, contradiction of the data of the influence oscillations on the cutting process is explained by this.

\section{References}

1. A.A. Voronin, STIN, 11, 15 (2003) 
2. V.N. Poduraev, Cutting with Vibrations (Mashinostroenie, Moscow, 1970)

3. D. Kumumbe, Vibration cutting, (Mashinostroenie, Moscow, 1970)

4. A.I. Markov, V. Mach., 10, 19 (1996)

5. B. M. Brzhozovsky, Ultrasonic technological processes and the equipment in machine and instrument making (Izd. SGTU, Saratov, 2009)

6. I. G. Zharkov, Vibrations when processing by the tool blade (Mashinostroenie, Leningrad, 1986)

7. I. G. Tkachenko, V. Mach., 2, 48 (2010)

8. Agapov S. I. Increase in efficiency of machining by application of ultrasound (Izdat. SNC, Sam., 2009)

9. S. I. Agapovof, V. Mach., 4, 66 (2008)

10. V.K. Astashev, Problems of mechanical engineering and reliability of machines, 359 (1967)

11. V.A. Kudinov, Dynamics of Machines (Mashinostroenie, Moscow, 1967)

12. V.L.Veits, D.V.Vastil'kov, Stanki Instrum., 6, 9 (1999)

13. Y.I. Gorodetsky, V. of Lobachevsky Un. of N. Nov, 2, 69 (2001)

14. V.L. Zakovorotny, A.A. Gubanova, A.D. Lukyanov, STIN, 4, 32 (2016)

15. S.A. Voronov, I.A. Kiselev, Mech. Engine. and Engine. Ed., 2 (51), 9 (2017)

16. A. M. Gouskov, S. A. Voronov, H. Paris, S. A. Batzer, Commun. Nonlin. Sci. Numer. Simul. 7, 207 (2002)

17. Y-C. Kao, N-T Nguyen, M-S. Chen, ST. Su. The Inter. Journal of Adv. Manuf. Tech., 1,1793 (2005)

18. J. Warminski, G. Litak, M. P. Cartmell, R. Khanin, M. Wiercigroch, Journal of Sound and Vibration, 259 (4), 917 (2003)

19. G. Stepan, Nonlin. Dyn.of Mat. Proc. and Manuf., 471152935, 165 (1998)

20. G. Stepan, T. Insperge, R. Szalai, International Journal of Bifurcation and Chaos, 15, 9, $2783(2005)$

21. V.L. Zakovorotny, A.D. Lukyanov, A.A. Gubanova, V.V. Khristoforova, Journal of Sound and Vibration, 368, 174 (2016)

22. V.L. Zakovorotny, V.E. Gvindjiliya, News of h. educ. Inst. App. nonlinear dyn., 25, 40 (2017)

23. V.L. Zakovorotny, T.H. Pham, Vestnik of DSTU. 6, 97 (2013)

24. V.L. Zakovorotny, V.E. Gvindjiliya, STIN, 4, 35 (2018)

25. V.L. Zakovorotny, V.E. Gvindjiliya, Izvestiya vuzov. The North Caucasus region. Technical science, 4, 67 (2016)

26. 25.V.L. Zakovorotny, V.E. Gvindjiliya, Vestn. Donsk. Gos.Tekh. Univ., 17, 35 (2017)

27. V.K. Astashev, VNTR, 2, 12 (2007)

28. V.K. Astashev, V.I. Babitsky, Ultrasonic, 6, 89 (1998)

29. V. K. Astashev, N. A. Andrianov, V. L. Krupenin, VNTR, 1, 3 (2017)

30. V.L. Zakovorotny, D.T. Pham, S.T. Nguyen, M.N. Ryzhkin, Vestnik of DSTU, 11, 137 (2011)

31. V.L. Zakovorotny, D.T. Pham, S.T. Nguyen, M.N. Ryzhkin, Vestnik of DSTU, 11, 301 (2011) 